\title{
English Teaching Evaluation Combined with End-User Computing and Neural Network
}

\author{
Qingyan Tan \\ School of Foreign Language, Hubei Polytechnic University, Huangshi 435003, China \\ Correspondence should be addressed to Qingyan Tan; 210044@hbpu.edu.cn
}

Received 6 January 2022; Accepted 31 January 2022; Published 23 February 2022

Academic Editor: Hasan Ali Khattak

Copyright (c) 2022 Qingyan Tan. This is an open access article distributed under the Creative Commons Attribution License, which permits unrestricted use, distribution, and reproduction in any medium, provided the original work is properly cited.

\begin{abstract}
A neural network model and English teaching evaluation of university with end-user computing are the focus of this paper. The main research contributions are as follows: (1) Propose an ADA-BP neural network. It applies the adaptive learning rate as well as the momentum term to promote the BP network. Experiments show that the model solves the problems of the existing methods and models such as difficulty in determining weights, prone to overfitting, slow convergence, and prone to local minimums, which verifies the effectiveness in evaluating college English teaching. (2) Propose the DA-SVR network. The model has multiple hidden layers and performs multiple feature conversions to optimize the network. The output layer of the model uses SVR as a predictor to achieve evaluation. (3) Deploy the designed evaluation network on the server, and end users can use the API interface to directly access and test the model functions. Massive experiments have verified the effectiveness of the designed method.
\end{abstract}

\section{Introduction}

The provision of English language instruction in postsecondary institutions is an essential service to the general public. When it comes to higher education reform and society's needs, the importance of teaching quality evaluation has grown in prominence. College English curriculum teaching has been stressed by the Ministry of Education's Dept. of Higher Education in a document that stresses the importance of reforming college curriculums, teaching techniques, and modes of delivery. Teachers are not the only ones who benefit from receiving feedback on their teaching methods. Teaching quality management and assurance is an excellent approach for students to change their strategies, enhance processes, and increase efficiency in their learning. However, the need for high-level, high-quality, and highskilled individuals in the domestic and the overseas markets has expanded substantially in recent years, even though colleges and universities have achieved some progress. It is essential that colleges and universities enhance the quality of teaching, strengthen the evaluation of teaching quality, and innovate and reform their talent training plans if they are to satisfy the needs of society and become more competitive professionals. A long time ago, there was a lack of theoretical and practical research on English teaching and teaching quality evaluation in higher education that has severely limited the growth of the innovative English education and teaching at universities. In order to increase the quality of college students to meet international talent needs, it is critical to improve the research and study of associated ideas and practices [1-5].

The teaching quality evaluation system guides the teaching content and practical activities to a large extent, and can find the problems and explore the solutions in a targeted manner. Looking at the research and exploration of English teaching evaluation, it is found from the perspective of theoretical research. On the one hand, the guiding documents formulated for teaching evaluation only involve basic requirements of English education, but do not make a detailed explanation of the core elements. On the other hand, a high proportion of research results of various researches done by domestic and foreign research institutions and scholars are based on the evaluation of English teaching. The English teaching background for foreign universities is very different from our country, and the research results lack the particularity and pertinence of the English teaching 
evaluation of Chinese universities. Traditional teaching evaluation guiding the ideology of light basic courses and heavier professional courses have a significant impact on research practice. Summative and formative evaluations of English education are still widely used in colleges and universities, with summative evaluations taking precedence over formative evaluations. Ignoring changes in the evaluation elements during the educational process is not a good way to encourage innovative thinking and personal development of evaluation objects [6-10].

On the other hand, the number of global end users has experienced rapid growth in the past ten years. GSMA pointed out in the 2014 Mobile Economy Report that as of 2013, the number of global terminal devices connected to 6.9 billion, and it is expected that this number will continue to increase in the next few years. At present, user terminals have become a popular computing platform, and their performance is more powerful. High-end mobile terminals are equipped with high-speed wireless interfaces, G-bit memory, $\mathrm{GHz}$-speed processors, and various sensors. Users have higher and higher expectations for the terminal, and they hope to run various applications with various functions on the user terminal. As a rising topic in recent years, enduser computing has received extensive attention from researchers. This allows end users to have their own processing terminals and can perform various system operations on their own terminals. End users can work efficiently on any supported end device while improving IT agility and organizational security. Resources can be expanded or reduced as needed to provide the team with the resources needed without the need to deploy and operate infrastructure [11-15].

Based on the two topics of college English teaching evaluation and end-user calculation, this work has conducted a lot of research. The contributions of this work are: (1) Propose an ADA-BP model for low-dimensional data. It applies the adaptive learning rate as well as the momentum term to promote the BP network. (2) For high-dimensional data, the DA-SVR network is proposed. SVR is used as a predictor to perform prediction, which realizes the mapping of nonlinear relationships to high-dimensional spaces. (3) Deploy the designed model on the server, expose the API interface, and end users can directly access and evaluate the quality for English teaching. This constitutes a complete evaluation system based on end-user computing and neural networks.

\section{Related Work}

Construction and optimization of the teaching evaluation system: literature [16] proposed the term education evaluation and designed a variety of evaluation methods. Research on the development of teaching evaluation showed that Western evaluation paid much attention to learner selfevaluation. It emphasized that learners were the main body, and the evaluation methods adopted were more diversified, relatively scientific, and reasonable [17]. The teaching evaluation system of the University of Washington, the teacher curriculum evaluation of the University of Arizona, and the teaching evaluation and individual development system of Kansas State University were all representative evaluation index systems [18]. Western countries had developed some relatively mature teaching evaluations. For example, Cisco had developed an advanced system. The company continuously improved plans and courses based on these evaluation data. Literature [19] had developed a learning performance evaluation system by fusing four computational intelligence theories, K-Means clustering algorithm, grey association theory, fuzzy reasoning, and fuzzy association rules. In addition, the research on teaching evaluation also included the emotional mining of students [20], and the extraction and analysis of teaching evaluation index scores [21]. Literature [22] carried out the earliest evaluation for classroom teaching quality. Since then, research on teaching evaluation had gradually started. The evaluation system develops evaluation indicators. Literature [23] introduced the NSSE questionnaire from the United States, and it was Chineseized to form a questionnaire for college students' learning input. Researchers had conducted an empirical analysis on the NSSE after Sinicization, which showed the questionnaire had ideal measurement reliability [24]. In early stage of the establishment of the indicator system, the pursuit of comprehensiveness of indicators often caused problems such as too many indicators and overlap between indicators, which led to the distortion of the indicator structure and caused people to question the objectivity of the evaluation. The basic methods to eliminate index correlation included the principal component analysis, factor analysis, entropy method, and the correlation coefficient calculation [25]. Literature [26] used the correlation coefficient analysis to judge the correlation between evaluation indicators.

Using neural network technology to evaluate educational content: teaching evaluation was no longer a single qualitative evaluation, but rather a blend of qualitative and quantitative evaluations as new technologies. For the quantitative analysis of varied data materials, however, a solid data model was generally required. The current mainstream teaching evaluation contained the weighted average method, the analytic hierarchy process [27], a fuzzy comprehensive evaluation [9], a neural network [28], and the Markov chain [29]. Fuzzy evaluation of the teaching quality based on the analytic hierarchy process (AHP) and literature [30] was a relatively scientific quantitative technique, which improved both the scientific standard and the reliability of the results. Using the rough set theory, decision trees, and association rule algorithms to examine aspects influencing teaching quality were all part of the research into incorporating a neural network technology into the college and university teaching evaluation systems.

Artificial neural networks were used in the literature [31] to represent instructional evaluation. Studies [32] used the artificial neural network theory to evaluate ethnic colleges and universities for their teaching quality, establishing associated mathematical models, completely quantifying indicators, and building BP models to get at a more reasonable evaluation conclusion. Based on wavelet neural networks, literature [33] had developed a mathematical model of teaching quality rating that is more accurate. 


\section{ADA-BP Neural Network for English Teaching Evaluation}

The section addresses the shortcomings of the existing methods and models of English teaching evaluation in dealing with small-scale, low-dimensional evaluation datasets. This work proposes an ADA-BP network. This model introduces the adaptive learning rate as well as the momentum term to promote the BP network.

\subsection{Advantages of the BP Network Applied to English Teaching} Evaluation. This evaluation is an important content, which is important to improving the quality of English teaching, and strengthening the standardization and scientification of the teaching management process. Teaching activities are a dynamic process that combines teaching and learning. The nonlinear relationship between evaluation indicators as well as teaching quality evaluation results is difficult to express with a specific and accurate mathematical analysis. However, many methods have some shortcomings in the methods and models. For example, the analytic hierarchy process finds it difficult to determine weight when there are too many evaluation indexes. Fuzzy comprehensive evaluation is too subjective and random, which can easily cause distortion of the evaluation results. The grey relational analysis is a linear analysis, and its ability to deal with nonlinear problems is limited. The decision tree algorithm easyily generates complex structures in the training data, which is easy to cause overfitting. The parameter optimization method of support vector machine has some shortcomings, such as slow optimization speed.

The BP network is widely used in neural networks as illustrated in Figure 1. It has a strong nonlinear mapping ability and can provide a solution for English teaching evaluation.

Therefore, applying the BP network to teaching quality evaluation can not only solve the problem between the qualitative and nonqualitative evaluation index system, but can also solve the issue for establishing complex data analysis formulas and avoid the interference of human factors to make the evaluation results more accurate. Some issues remain in the training process, including a slow convergence rate and the tendency to slip into a local minimum that can be difficult to avoid. A new model is proposed in this section in light of the inadequacies of the BP network.

3.2. The ADA-BP Network. Since English teaching evaluation is a multi-level and nonlinear problem, present evaluation methods have issues such as difficulties in determining the weight of each objective, strong subjectivity, and prone to overfitting. It is also susceptible to difficulties such as delayed convergence and the tendency to slip into a local minimum when using the conventional BP neural network. The ADA-BP model is presented in this section. To speed up the BP network's gradient descent approach, the model introduces adaptive learning rates and momentum

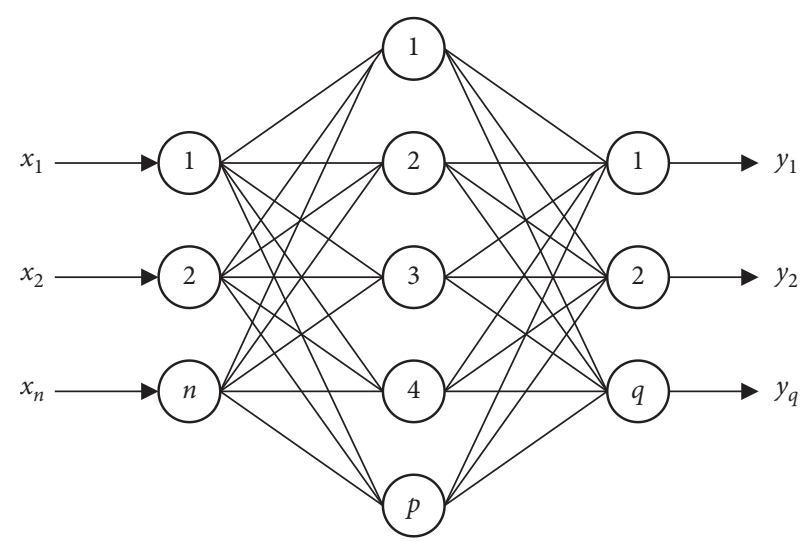

FIGURE 1: Structure of the BP network.

terms. The network structure is optimized to assure the model's stability.

A three-layer BP network can satisfy nonlinear mapping from a-dimensional to $\mathrm{b}$-dimensional functions. Therefore, this paper designs a three-layer BP network for English teaching evaluation. The number of neurons is determined by the dimension of the input sample and the dimension of the output. However, the selection of the hidden layer is more complicated and there is no good theoretical guidance. Based on experience, this work uses the following methods to determine neurons:

$$
d=\sqrt{a+b}+c,
$$

where $a$ and $b$ are number of neurons in the input layer and the output layer and $c$ is a constant.

The BP network has a fixed learning step length, or learning rate. The network structure will become unstable and oscillate if the learning rate is excessive. The network will converge more slowly if the learning rate is low. As a result, optimizing the overall network topology for problem-specific learning is difficult. In order to continuously correct the weights and the thresholds between the linked layers, an adaptive learning rate can adjust the learning rate based on the changes of the network error. Assuming the initial learning rate is $\operatorname{lr}(0)$, the network error is $E(k)$ and the learning rate change is

$$
\operatorname{lr}(k+1)=\left\{\begin{array}{l}
\alpha \operatorname{lr}(k), \quad E(k+1)<E(k) \\
\beta \operatorname{lr}(k), \quad E(k+1)>E(k), \\
\operatorname{lr}(k), \text { others }
\end{array}\right.
$$

where $\alpha$ and $\beta$ are the weights.

The convergence speed can be increased by adaptively altering the learning rate throughout the error back propagation phase. Only changes in the gradient descent direction at the current time $t$ are considered, and the gradient direction before the current time $t$ is not considered, which will lead to a model that readily falls into a local minimum due to instability. With the momentum element added, the weight of the error back propagation mechanism can be modified to dampen the effect of the errors. The formula is given as follows: 


$$
\Delta w(k+1)=\mu \Delta w(k)-\operatorname{lr} \frac{\partial E(k+1)}{\partial w(k+1)},
$$

where $\mu$ is the momentum term.

3.3. ADA-BP in English Teaching Evaluation. The processing flowchart of the ADA-BP used in English teaching evaluation is shown in Figure 2.

The model changes the learning rate with a comparison of the current error to the prior error and adds a momentum term to dampen the model to prevent oscillations and assure stability. As part of the gradient descent approach of BP, introduce adaptive learning rates and momentum terms. As going through the training process, make adjustments to the model's network topology, such as the number of hidden layer neurons, adaptive learning rate, as well as momentum items. When the evaluation result reaches the set condition, exit training and retrieve the final model that has been trained at this moment. Finally, input the test dataset for verification to show the proposed model has good accuracy and advantages in tackling English teaching evaluation issues.

\section{DA-SVR Neural Network for English Teaching Evaluation}

The ADA-BP network has certain advantages in processing low-dimensional data of college English teaching evaluation. However, when faced with a large-scale dataset with complex, high-dimensional, and nonlinear relationships, the computing power, the predictive power, and the modeling expression power are limited because it contains only one hidden layer. In order to solve the application problems on large-scale datasets with complex, high-dimensional, and nonlinear relationships, this section proposes the DA-SVR deep neural network model. The deep denoising auto encoder of the DA-SVR model is a stack of multiple denoising auto encoders. It contains multiple hidden layers. Noise is added to enhance the robustness. The Adam algorithm is introduced to improve the computing power. After multiple feature conversions, the error is minimized. The output layer utilizes SVR as a predictor.

4.1. Deep Denoising Auto Encoder. Deep denoising auto encoder is a neural network, which is composed of multiple, stacked denoising auto encoders. Among them, the denoising auto encoder adds noise to the original input with an auto encoder to prevent overfitting during the training. The model obtained after training has strong robustness.

Denoising the input dataset is the initial step in the training process of the deep denoising auto encoder. With a high degree of probability, certain input nodes are set to 0 . This is the standard processing procedure. Then, using the first denoising auto encoder, convert the input layer's data features to the hidden layer using the denoised dataset. The second auto encoder takes the input feature vector from the hidden layer of the first denoising auto encoder. It's a good idea to keep repeating this process, utilizing the previous denoising auto encoder's hidden layer output as the input feature vector for the next denoising auto encoder. Data feature conversion is completed via unsupervised layer-bylayer training of the original input data through all hidden layers after noise denoising has been applied. The original dataset and the rebuilt dataset are used to calculate errors through a function that takes both into account. Use the BP algorithm to propagate the mistake to all the deep noise denoising auto encoders, then reduce the option weight and threshold to finish the reconstruction of the original input dataset and produce the feature. The structure diagram of this part of the deep denoising auto encoder is illustrated in Figure 3.

The Adam algorithm is applied for unsupervised training. The neurons in the output layer of the deep denoising auto encoder in the figure are virtual representations, and the actual output layer is a classifier or predictor as a supervised output classification or regression prediction. By adjusting the parameters of the supervised output layer, the accuracy of the classification or prediction can be improved.

4.2. Support Vector Regression. Mathematical expressions of college English teaching are problematic since there are so many evaluation indexes and a complex nonlinear relationship between them and the evaluation outcomes of teaching quality. Support vector regression has a good capacity to fit nonlinear functions. As a result, the output layer of the deep neural network model in this section is predicted using support vector regression as the predictor.

SVR can be separated into linear and nonlinear regression, depending on whether it is applied to a high-dimensional space. This section focuses on the nonlinear regression of support vector regression since the evaluation of college English education is a difficult nonlinear problem. Using the nonlinear regression of support vector regression, it is possible to map the complex nonlinear relationship to the high-dimensional space and then realize the linearized relationship equivalent to the low-dimensional space. Setting up a nonlinear mapping function first for the dataset $S$ that cannot be linearly separated in the original space is the first step. Make sure that the mapped data have good linear regression characteristics in the feature space as well. For the linearized representation of nonlinear issues, first perform linear regression in the feature space, and then return to the original space for the linearization. Given a kernel function $k\left(x_{i}, x\right)$, the expression for constructing a nonlinear function is given by

$$
f(x)=\sum_{i=1}^{S}\left(a_{i}-a_{i}^{*}\right) k\left(x_{i}, x\right)+b,
$$

where $a_{i}, a_{i}^{*}$ and $b$ are the equation parameters and $x_{i}$ and $x$ are the input data.

The frequently used kernel functions are as follows: linear function, polynomial function, radial basis function, and sigmoid function, among which $\sigma$ is a parameter: 


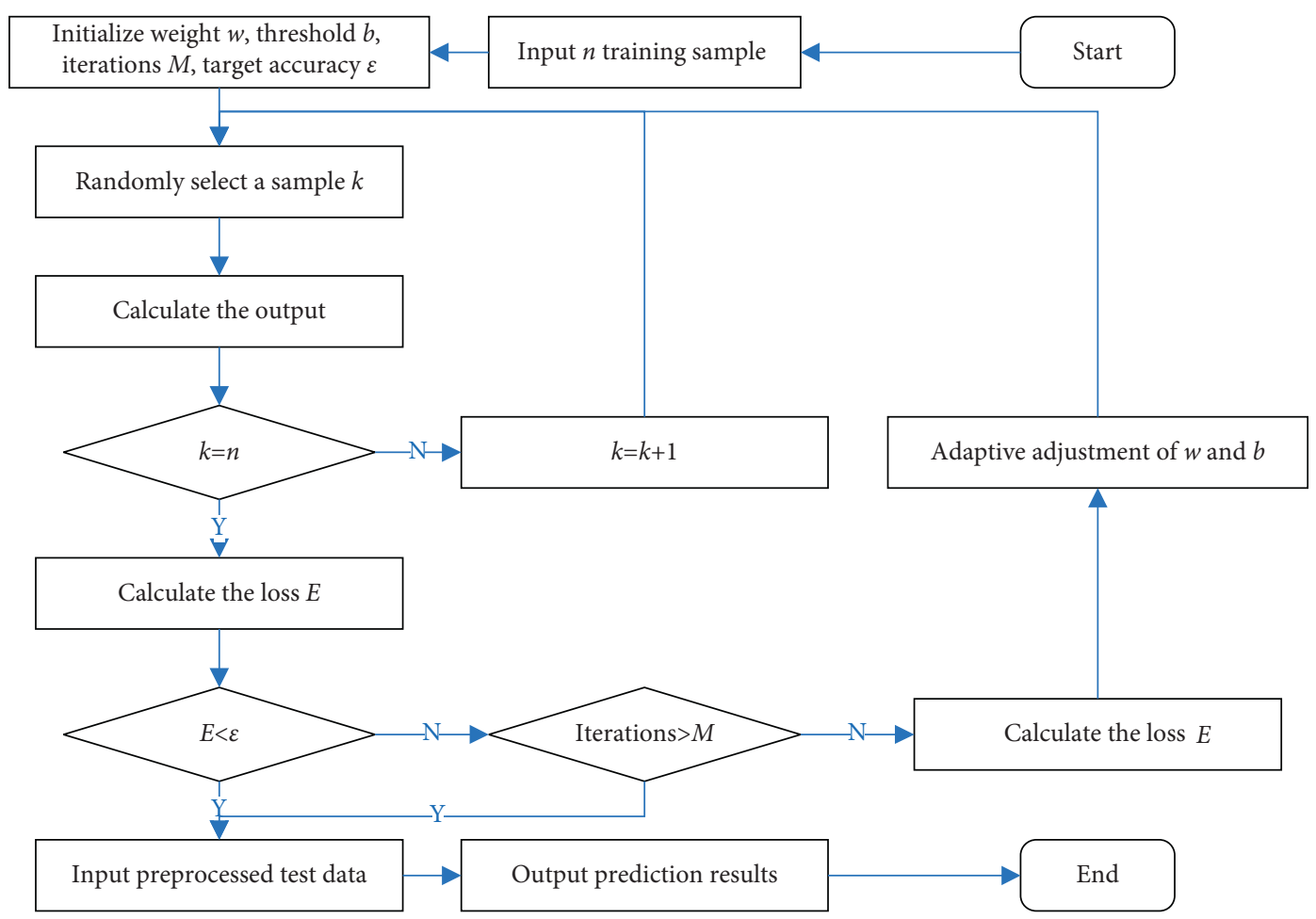

FIgURE 2: The flowchart of the ADA-BP model applied to English teaching evaluation.

$$
\begin{aligned}
& k\left(x_{i}, x\right)=x^{T} x_{i}, \\
& k\left(x_{i}, x\right)=\left(1+x^{T} x_{i}\right)^{\sigma}, \\
& k\left(x_{i}, x\right)=\exp \left(\frac{-x-x_{i}^{2}}{\sigma^{2}}\right), \\
& k\left(x_{i}, x\right)=\tanh \left(x^{T}, x_{i}\right)+\sigma .
\end{aligned}
$$

4.3. The DA-SVR Network. In order to deal with big datasets or handle complex nonlinear problems, this section uses the DA-SVR networks. The unsupervised training layer is the deep denoising automated coding, and the prediction output layer is the support vector regression. Unsupervised training layer preprocessing is performed using Adam optimization, which utilizes first-order moment estimation and secondorder moment estimation to change the learning step length to improve the convergence speed and computing power. SVR is utilized in the output layer to achieve a linearized relationship similar to the low-dimensional space in the set high-dimensional space. Unsupervised layer-by-layer learning and training is performed using a deep denoising auto encoder on the original input dataset. To get the original input dataset's feature vector, minimize the error between the original dataset and the training data. To forecast the final layer of the model, utilize support vector regression, and use the original dataset's feature vector as the input for support vector regression. The structure of the DASVR network is illustrated in Figure 4.
The DA-SVR deep neural network model mainly has the following two processes: (1) Unsupervised training layer by layer. Before the original input data is input to the DA-SVR network model training, there will be some noise in the original input data that cannot be cleaned. To prevent the occurrence of overfitting, features of the original input data will be set to 0 according to a certain proportion, and the noise will be reduced to increase robustness. The unsupervised training process is mainly to use the deep denoising auto encoder for training, and the denoising processed data enters the first DA for training. The obtained output is utilized as the input feature for the second DA, and so on to complete the training of all Das. In fact, each DA is equivalent to a hidden layer. Calculate the error, and introduce Adam to optimize the error to minimize it. (2) Supervised fine-tuning process. The feature vector of the last hidden layer of the auto encoder is utilized as the input feature for the last output layer. The final output layer uses SVR as the predictor. In this supervised process, the relevant parameters of SVR are tuned to improve the prediction accuracy and efficiency.

4.4. DA-SVR in English Teaching Evaluation. The model contains multiple hidden layers. For complex nonlinear issue as well as large-scale dataset issue, the deep structured neural network model can perform calculations and modeling well. Therefore, the DA-SVR model is used to solve the teaching evaluation. Assuming the weight matrix is $W$ and the threshold matrix is $c, E n$ is a deep denoising auto encoder model. When the input dataset passes, its expression is as follows: 


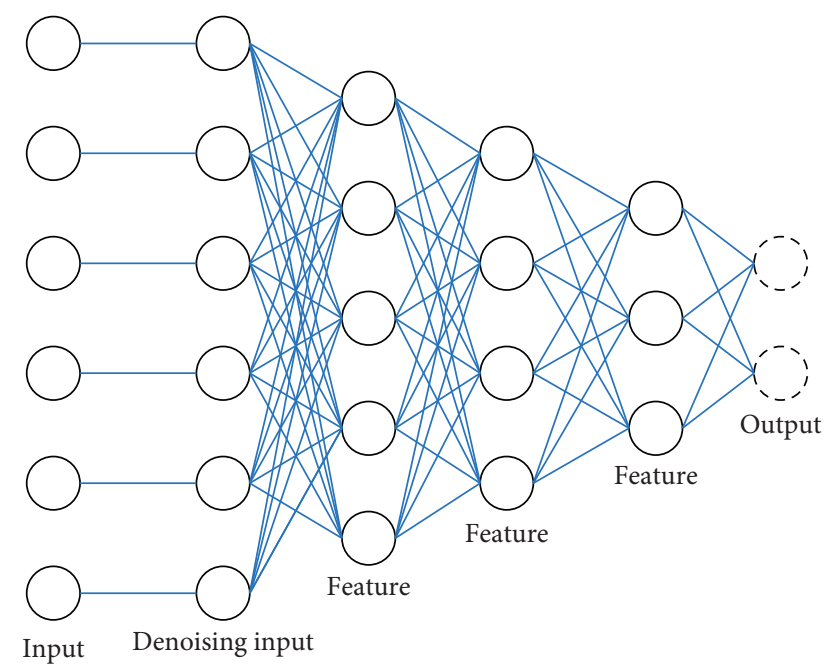

FIGURE 3: Deep denoising auto encoder structure.

$$
d=E n(W x+c)
$$

where $d$ is the output feature vector, and input into the SVR for prediction. Taking $f$ as the function of SVR, the expression is

$$
e=f(d)
$$

The flowchart of the DA-SVR model for evaluating college English teaching is shown in Figure 5.

To train the model stated in this section, the evaluation sample dataset is split into a training dataset and a test dataset. A stable and optimal model is obtained by altering the number of hidden layers of the model, optimization techniques, and other parameters, as well as relevant parameters of support vector regression. To test the model's ability to evaluate instruction quality, it needs an input test dataset.

\section{Combination with End-User Computing and Neural Network}

With the development of terminal equipment and related technologies, terminal user computing has become mainstream. Therefore, this work combines end-user computing and neural networks to build an English teaching evaluation system that combines end-user computing and artificial neural networks. The schematic diagram of the system is illustrated in Figure 6.

The joint system designed in this article is a C/Sstructured English teaching evaluation system. First, use the relevant data of English teaching to train an English teaching evaluation network, which is the ADA-BP or the DA-SVR network model designed by this work. Then, deploy the trained model on the remote server, which provides an API call interface to the outside. After that, end users can use various mobile terminals ( $\mathrm{PC}$, tablet, and mobile phone) to call this API interface to complete the evaluation of English teaching.

\section{Experiments and Discussions}

6.1. Evaluation on the ADA-BPNetwork. The data used in the experiment in this section are obtained from the records of a university educational administration system, and the obtained data are normalized and preprocessed as the input feature. The structure of the model given in the experiment is three layers. The training dataset contains 1551 samples, and the test dataset contains 206 samples. Each sample contains 10 different evaluation indicators; the specific distribution is shown in Table 1. The mean square error (MSE) and prediction accuracy are used as evaluation performance comparison indicators.

The improved gradient descent approach is more effective compared to the classic gradient descent method for determining the insertion of adaptive learning rates as well as momentum terms into the BP neural network's training. To get a stable network, input the training dataset into the model, and then input the test dataset for verification, and utilize two separate methods as the training function. How many times the model has been trained is compared to how long it has been running, as well as how accurate it is at predicting the actual results. Table 2 shows the results of the experiment, in which an average of 10 runs is utilized as the final result. AL is adaptive learning rate. MT is momentum term.

An adaptive learning rate as well as momentum term improved the gradient descent approach, which can improve a model's convergence speed when utilized as a training function, according to the results. There is also a significant reduction in errors, which is more effective in terms of accuracy as well as training iteration.

To determine the number of neurons for the hidden layer, we use formulas to determine the range, and use trial and error experiments to determine the optimal number. When the number of neurons for the hidden layer of the model changes, the change curve of the MSE and prediction accuracy is shown in Figure 7.

When the number of neurons in the hidden layer is 10 , the MSE and prediction accuracy of the model perform best at this time. Therefore, considering the overall performance indicators, choosing the number of hidden layer neurons as 10 is more conducive to subsequent experiments.

In order to ensure the stability of the network, a momentum term is introduced to punish the variation of the learning rate and avoid model instability. This part selects momentum items from $[0.5,1.0]$ for experiments. When different momentum items are added to the model training, the MSE and prediction accuracy obtained by the model during the verification test are shown in Figure 8.

When the momentum term is selected as 0.7, the MSE and prediction accuracy are the best. In view of this, 0.7 is selected as the momentum term of the model.

To verify the effectiveness of the model in evaluation of college English teaching, logistic regression, SVM, decision tree, and traditional BP networks are used as comparison methods to compare with the ADA-BP method designed in this work. The experimental results are shown in Table 3. 


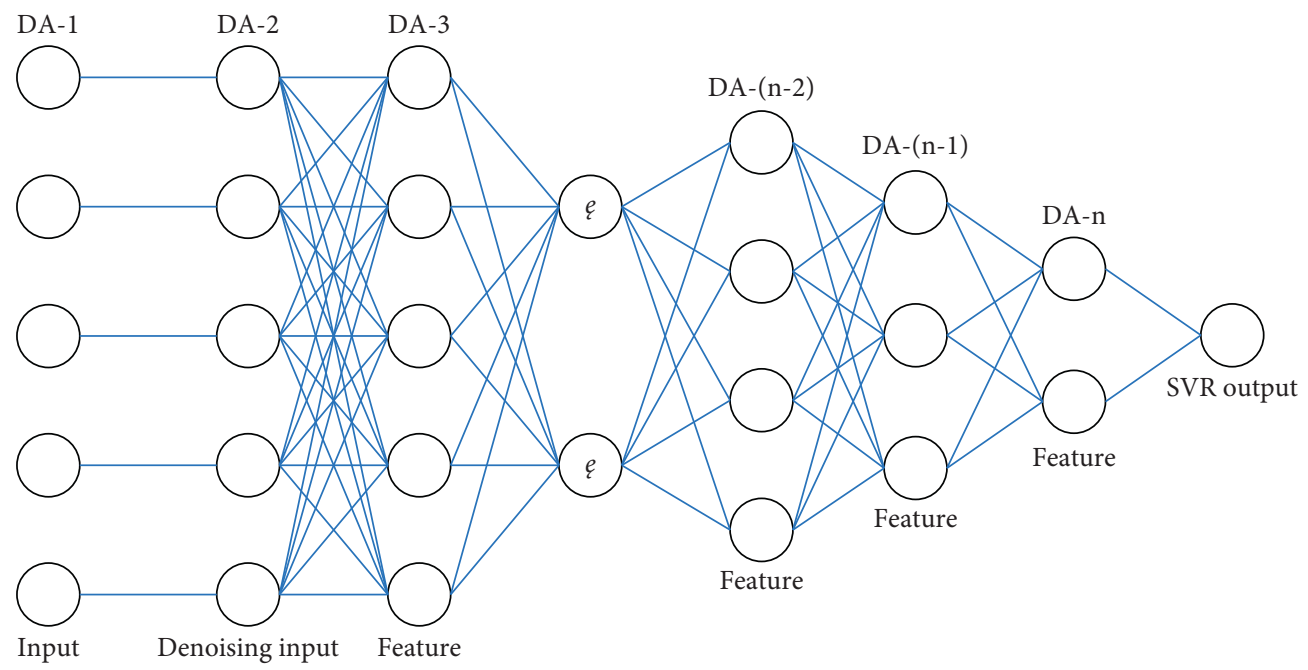

FIgURE 4: The structure of the DA-SVR.

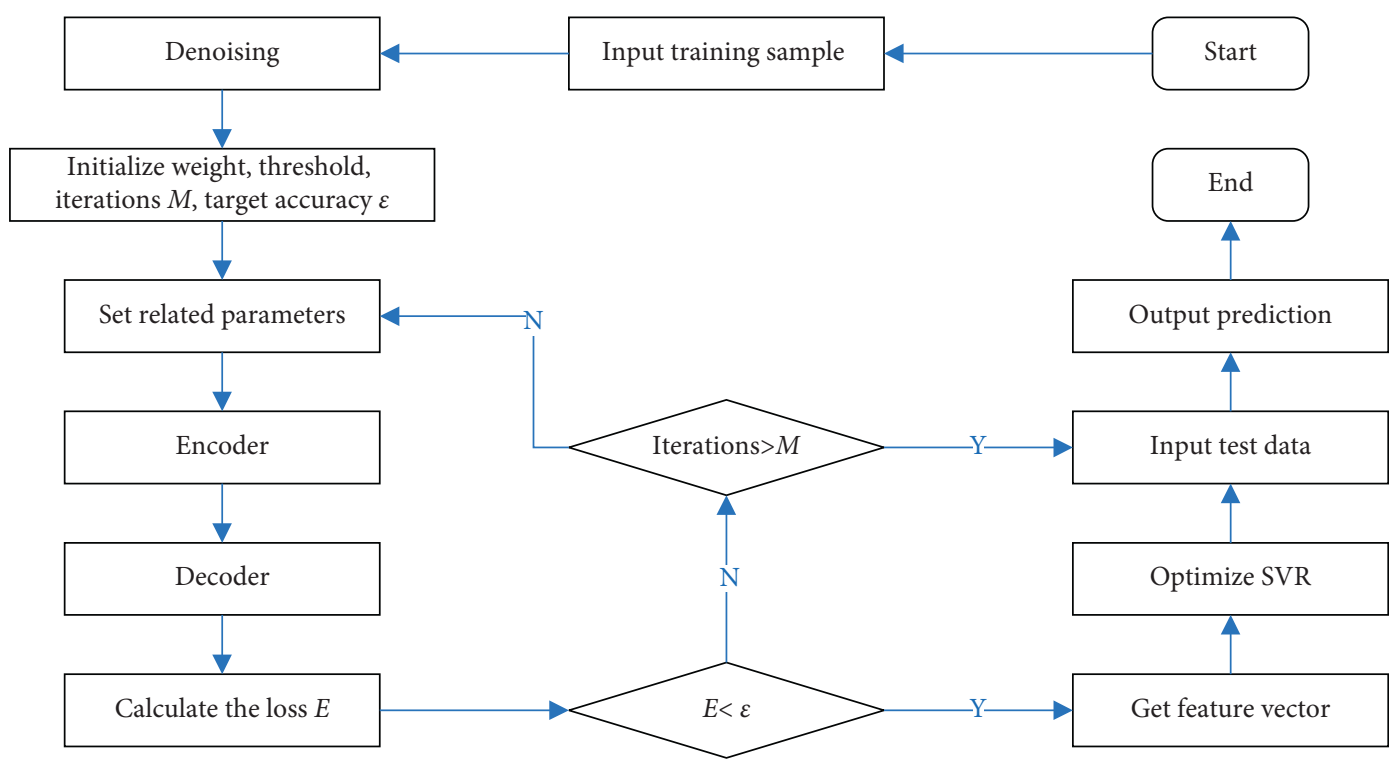

Figure 5: The flowchart of the DA-SVR model applied to English teaching evaluation.

The ADA-BP model proposed in this section not only overcomes the difficulty of determining the index weight, but also solves the problems of overfitting, slow convergence, and falling into local minimums. It also improves the accuracy of the evaluation prediction, thus verifying the effectiveness of the model in English teaching evaluation.

6.2. Evaluation on the DA-SVR Network. The DA-SVR model designed in this work can process higher-dimensional nonlinear features. In order to obtain higher-dimensional features, first expand the English teaching evaluation indicators. In addition to the indicators in Table 1, the indicators in Table 4 are also used.

To determine the appropriate number of hidden layers of the DA-SVR, set the number to be selected between 1 and 5 . Adam is embedded as an optimization algorithm. The features of the evaluation sample dataset are input into the DA-SVR model. The MSE and prediction accuracy of the network are shown in Figure 9.

After training, the reconstructed output data have the lowest error and the network performs best when there are two hidden layers in the DA-SVR model, which is the default.

This part picks from 21 to 25 neurons for each hidden layer of the DA-SVR model. This technique uses the Adam algorithm as an optimization method for unsupervised learning and training, which has two hidden layers. Adjust the amount of neurons in the hidden layer based on the evaluation sample dataset. The experimental result is illustrated in Figure 10.

It can be seen that when each hidden layer of the DASVR model is selected as 22 , the error between the output obtained by reconstruction as well as the original data are the best, and the test performance is the best. 


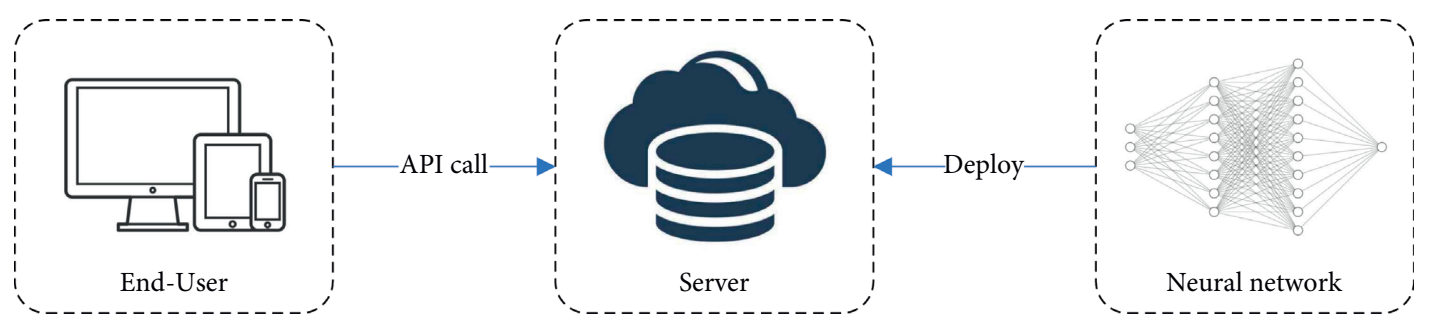

FIgURE 6: English teaching evaluation with end-user computing and neural networks.

TABLE 1: English teaching evaluation index.

\begin{tabular}{lc}
\hline Number & Index \\
\hline$X_{1}$ & Teaching appeal \\
$X_{2}$ & Listen to opinions \\
$X_{3}$ & Pay attention to methods \\
$X_{4}$ & Clear thinking and strong inspiration \\
$X_{5}$ & Teaching with students' aptitude \\
$X_{6}$ & Heuristic teaching \\
$X_{7}$ & Solve student's confusion \\
$X_{8}$ & Learn to analyze independently \\
$X_{9}$ & Answer questions seriously \\
$X_{10}$ & Correct homework in time \\
\hline
\end{tabular}

TABLE 2: Comparison of results of different training functions.

\begin{tabular}{lcccc}
\hline Method & MSE & Accuracy $(\%)$ & Train time & Iteration \\
\hline SGD & 61.7 & 91.7 & 9.4 & 1000 \\
AL + MT & 26.3 & 95.3 & 0.7 & 156 \\
\hline
\end{tabular}
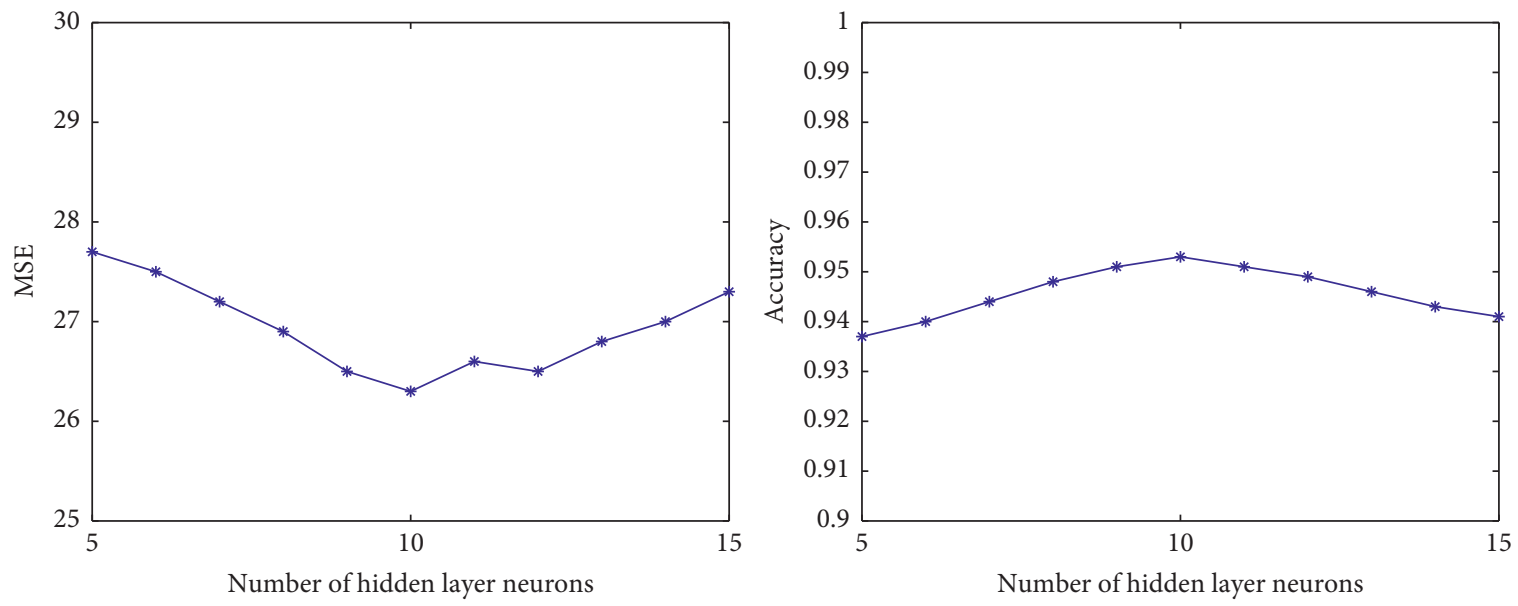

FIgURE 7: Evaluation on the number of hidden layer neurons.

The kernel function types of support vector regression are mainly the linear function (Liner), polynomial function (Poly), radial basis function (RBF), and Sigmoid function. This work compares the influence of different kernel functions on network performance, and the experimental result is illustrated in Figure 11.

Obviously, when the polynomial function (Poly) is used as the kernel function of support vector regression, the performance is better than the prediction effect of the other three functions; thus, the support vector regression kernel function is selected as the polynomial function.

To verify that the performance of the DA-SVR method in face of high-dimensional nonlinear feature modeling is better than that of the ADA-BP method, this work compares the two. The experimental result is illustrated in Figure 12.

Obviously, when facing high-dimensional nonlinear feature data, the designed DA-SVR method has better 

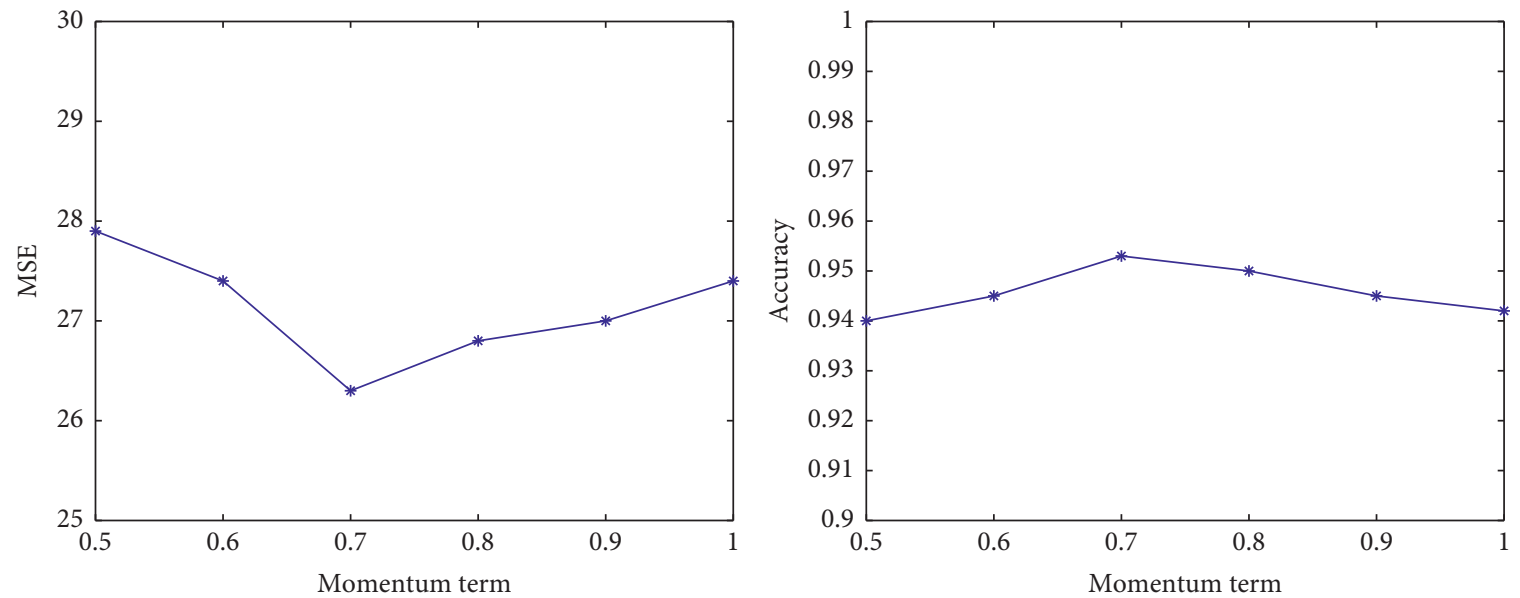

Figure 8: Evaluation on momentum item.

TABle 3: Comparison with other methods.

\begin{tabular}{lcccc}
\hline Method & MSE & Accuracy (\%) & Train time & Iteration \\
\hline Logistic regression & 87.4 & 88.2 & 0.2 & - \\
SVM & 81.9 & 89.9 & 0.2 & - \\
Decision tree & 76.2 & 91.7 & 0.4 & - \\
BP & 61.5 & 92.5 & 9.8 & 1000 \\
\hline ADA-BP (ours) & 26.3 & 95.3 & 0.7 & 156 \\
\hline
\end{tabular}

TABLE 4: Additional English teaching evaluation index.

\begin{tabular}{lc}
\hline Number & Index \\
\hline$X_{11}$ & Well prepared for teaching \\
$X_{12}$ & Teacher-student interaction \\
$X_{13}$ & Teaching content is substantial \\
$X_{14}$ & Active classroom atmosphere \\
$X_{15}$ & Reasonable teaching schedule \\
$X_{16}$ & Concise courseware production \\
$X_{17}$ & Clear course structure \\
$X_{18}$ & Clear and standard text and graphics \\
$X_{19}$ & Evaluation of student responses \\
$X_{20}$ & Classroom discipline is good \\
\hline
\end{tabular}
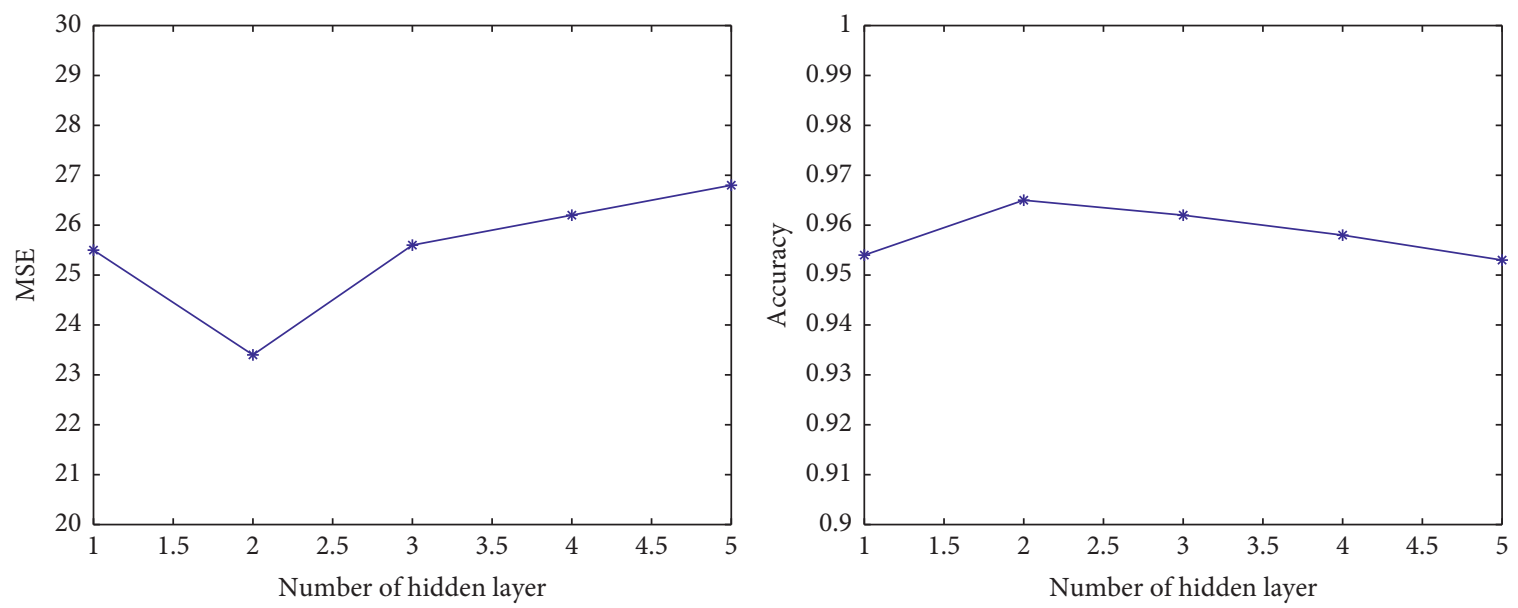

FIgURE 9: Evaluation on the number of hidden layers. 

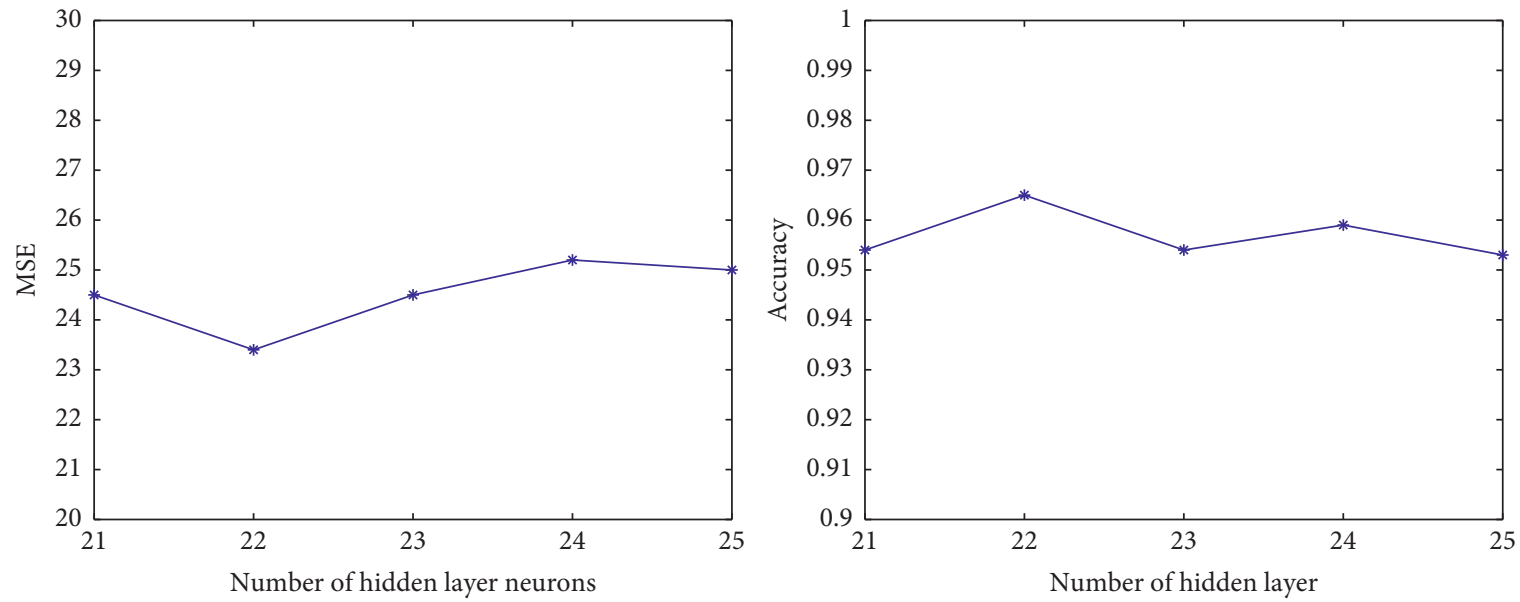

Figure 10: Evaluation on the number of hidden layer neurons.

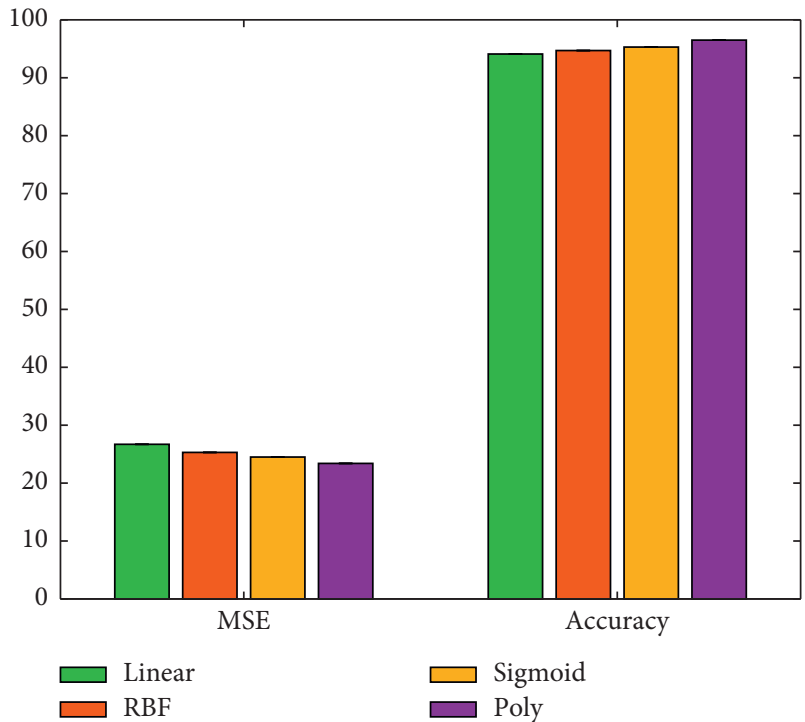

FIGURE 11: Evaluation on kernel function.

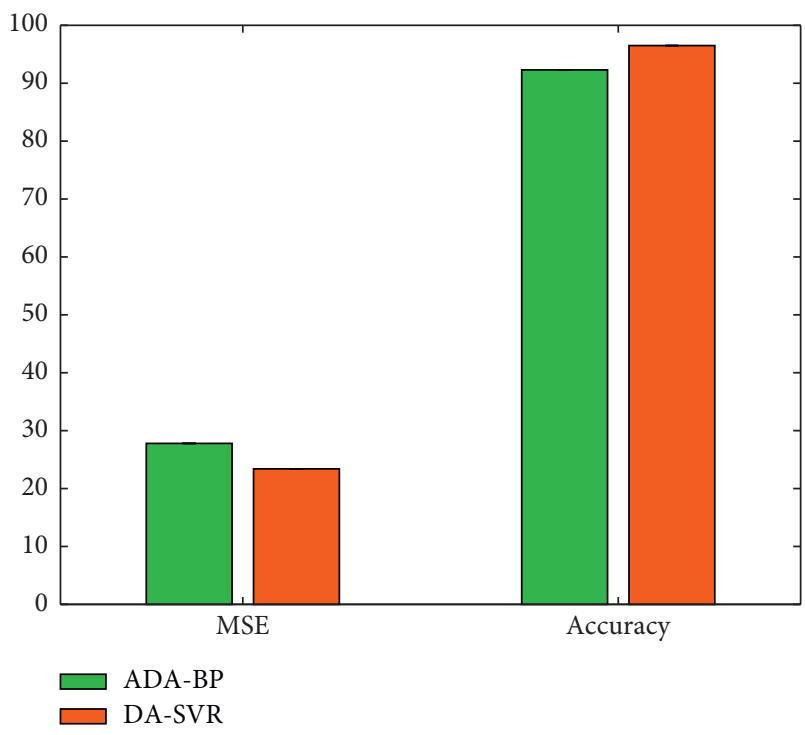

Figure 12: Comparison of the ADA-BP with the DA-SVR. performance than the ADA-BP method. This can also prove the effectiveness of the method designed in this work.

\section{Conclusion}

The appraisal of a college relies on the effective English instruction as a vital link in their overall instructional chain. As a result, there is a nonlinear relationship between the evaluation indicators and the quality of instruction, which is compounded by numerous factors. Traditional evaluation methods have problems such as too strong subjectivity and randomness, difficulty in determining the index weights, and slow optimization. Therefore, the main research contributions of this paper have the following two points: (1) For small-scale datasets, the existing methods and models have many problems. This work proposes the ADA-BP model, which mainly introduces the adaptive learning rate and momentum to improve the $\mathrm{BP}$ network. The results show the model can solve the issues existing in other methods, and predictive evaluation results are still optimal, thus verifying the effectiveness of the model in solving college English teaching evaluation. (2) For large-scale and highdimensional datasets, although the BP neural network has certain advantages in the evaluation of English teaching, its computing and modeling capabilities are limited. To solve it, this work proposes the DA-SVR model. This model is composed of deep denoising auto encoders and support vector regression. By comparing the model performance indicators, the effectiveness in the evaluation of college English teaching and the processing of large-scale datasets and its powerful computational modeling capabilities are verified. (3) Deploy the designed evaluation network on the server, and end users can use the API interface to directly access and test the model. In future research work, we will devote ourselves to designing models with higher performance and lighter weight to evaluate the quality of various teaching. If possible, we will also design a set of comprehensive and systematic evaluation indicators for teaching evaluation.

\section{Data Availability}

The datasets used during the current study are available from the corresponding author on reasonable request. 


\section{Conflicts of Interest}

The authors declare that they have no conflicts of interest.

\section{References}

[1] C. Huang, "Exploration and research of information technology in college English teaching," Journal of Physics: Conference Series, vol. 1648, no. 2, Article ID 022021, 2020.

[2] C. Meng-yue, L. Dan, and W. Jun, "A study of college English culture intelligence-aided teaching system and teaching pattern," English Language Teaching, vol. 13, no. 3, pp. 77-83, 2020.

[3] J. Cheng and L. Wei, "Individual agency and changing language education policy in China: reactions to the new guidelines on college English teaching," Current Issues in Language Planning, vol. 22, no. 1-2, pp. 117-135, 2021.

[4] Y. Du, "Study on cultivating college students' English autonomous learning ability under the flipped classroom model," English Language Teaching, vol. 13, no. 6, pp. 13-19, 2020.

[5] M. Bao, "Research on the new eco-construction of college English teaching in the data age," Studies in Literature and Language, vol. 20, no. 3, pp. 13-16, 2020.

[6] H. Li, "Improved fuzzy," Computational Intelligence, vol. 37, no. 3, pp. 1199-1216, 2021.

[7] H. Liu, R. Chen, and S. Cao, "Evaluation of college English teaching quality based on grey clustering analysis," International Journal of Emerging Technologies in Learning, vol. 16, no. 2, pp. 173-187, 2021.

[8] J. Pei, K. Zhong, J. Li, J. Xu, and X. Wang, "ECNN: evaluating a cluster-neural network model for city innovation capability," Neural Computing \& Applications, vol. 30, pp. 1-13, 2021.

[9] N. Li, "A fuzzy evaluation model of college English teaching quality based on analytic hierarchy process," International Journal of Emerging Technologies in Learning, vol. 16, no. 2, pp. 17-30, 2021.

[10] J. Guo and S. Yu, "Evaluation model of college English teaching quality based on big data analysis," IOP Conference Series: Materials Science and Engineering, vol. 750, no. 1, Article ID 012077, 2020.

[11] J. Sutton-Parker, "Determining end user computing device scope 2 GHG emissions with accurate use phase energy consumption measurement," Procedia Computer Science, vol. 175, pp. 484-491, 2020.

[12] F. Azzahrah, Y. A. P. Adian, and W. Budiarto, "Analisis Kepuasan Pengguna Mobile E-Health berdasarkan Metode End User Computing Satisfaction (Studi di 5 Puskesmas di Kota Surabaya)," Jurnal Kesehatan, vol. 11, no. 3, pp. 395-403, 2020.

[13] A. Rule, I. H. Goldstein, and M. F. Chiang, "Clinical documentation as end-user programming," in Proceedings of the 2020 CHI Conference on Human Factors in Computing Systems, pp. 1-13, Honolulu HI USA, April 2020.

[14] R. Munap, S. N. B. Ahmad, S. A. Hamid, and M. F. B. M. Talib Beg, "The influence of end user computing system (EUCS) on user satisfaction: the case of a logistic and courier service company," The International Journal of Social Sciences and Humanities Invention, vol. 5, no. 12, pp. 5103-5110, 2018.

[15] A. P. Sari and M. A. Syamsuddin, "Analisis faktor end user computing satisfaction terhadap kepuasan pengguna: studi kasus kantor pelayanan pajak madya balikpapan," Jurnal Pajak Indonesia (Indonesian Tax Review), vol. 1, no. 2, pp. 92-101, 2017.
[16] S. Ganesh and A. Binu, "Statistical analysis to determine the performance of multiple beneficiaries of educational sector using hadoop-hive," in Proceedings of the International Conference on Data Science \& Engineering, pp. 32-37, Kochi, India, August 2014.

[17] B. Kizlik, Measurement, Assessment, and Evaluation in Education, 2012.

[18] J. Fien, W. Scott, and D. Tilbury, "Education and conservation: lessons from an evaluation," Environmental Education Research, vol. 7, no. 4, pp. 379-395, 2001.

[19] C.-M. Chen, Y.-Y. Chen, and C.-Y. Liu, "Learning performance assessment approach using web-based learning portfolios for E-learning systems," IEEE Transactions on Systems, Man and Cybernetics, Part C (Applications and Reviews), vol. 37, no. 6, pp. 1349-1359, 2007.

[20] C. K. Leong, Y. H. Lee, and W. K. Mak, "Mining sentiments in SMS texts for teaching evaluation," Expert Systems with Applications, vol. 39, no. 3, pp. 2584-2589, 2012.

[21] H. Liu and Y. Xia, "Notice of retraction: teaching evaluation system based on association rule mining," in Proceedings of the Pacific-Asia Conference on Circuits, Communications and System, pp. 1-3, Chengdu, China, May 2011.

[22] G. G. Wang, "Bringing games into the classroom in teaching quality control," International Journal of Engineering Education, vol. 20, no. 5, pp. 678-689, 2004.

[23] T. Chambers, "What I hear you saying is: analysis of student comments from the NSSE," College Student Journal, vol. 44, no. $1,2010$.

[24] D. Tu, J. Shi, and F. Guo, "A metric study on NSSE-China," Fudan Education Forum, vol. 11, no. 1, pp. 54-62, 2013.

[25] J. Liu, "Research on processing method of evaluation index correlation," Digest of Management Science, vol. 1, no. 12, pp. 50-51, 2016.

[26] X. F. Xu and R. B. Xiao, "An approach of eliminating correlation of assessment-index," Systems Engineering-Theory \& Practice, vol. 22, no. 11, pp. 1-5, 2002.

[27] E. Thanassoulis, P. K. Dey, K. Petridis, I. Goniadis, and A. C. Georgiou, "Evaluating higher education teaching performance using combined analytic hierarchy process and data envelopment analysis," Journal of the Operational Research Society, vol. 68, no. 4, pp. 431-445, 2017.

[28] C. S. Galbraith, G. B. Merrill, and D. M. Kline, "Are student evaluations of teaching effectiveness valid for measuring student learning outcomes in business related classes? A neural network and bayesian analyses," Research in Higher Education, vol. 53, no. 3, pp. 353-374, 2012.

[29] V. P. Gurupur, G. Pankaj Jain, and R. Rudraraju, "Evaluating student learning using concept maps and Markov chains," Expert Systems with Applications, vol. 42, no. 7, pp. 33063314, 2015.

[30] X. Li, C. Luo, and Z. Liu, "A fuzzy comprehensive evaluation of the teaching quality of MOOC combined with AHP," Contemporary Continuing Education, vol. 34, no. 192, pp. 61-64, 2016.

[31] G. Zhang, J. Yin, L. L. Cheng, and C. R. Wang, "Machine learning based teaching quality evaluation," Advanced Materials Research. Trans Tech Publications Ltd, pp. 1451-1454, 2011.

[32] Z. Hua-ping, "Study on Bilingual Teaching Evaluation System of Colleges and Universities based on Factor Analysis Method," Journal of Taishan University, vol. 02, 2011.

[33] H. Li, "A teaching quality evaluation model based on a wavelet neural network improved by particle swarm optimization," Cybernetics and Information Technologies, vol. 14, no. 3, pp. 110-120, 2014. 